\title{
Mechanism of Sang-Ju-Yin on Hand, Foot and Mouth Disease Based on Network Pharmacology
}

\author{
Lianfang Xue, Hui Liu, Minghao Chen, Suishan Qiu* \\ Department of Pharmacy, The First Affiliated Hospital of Jinan University, Guangzhou, China \\ Email address: \\ xlf18666@163.com (Lianfang Xue), liu_ui@hotmail.com (Hui Liu), chen.minghao@hotmail.com (Minghao Chen), \\ 494490817@qq.com (Suishan Qiu) \\ ${ }^{*}$ Corresponding author
}

\section{To cite this article:}

Lianfang Xue, Hui Liu, Minghao Chen, Suishan Qiu. Mechanism of Sang-Ju-Yin on Hand, Foot and Mouth Disease Based on Network Pharmacology. International Journal of Chinese Medicine. Vol. 5, No. 1, 2021, pp. 6-13. doi: 10.11648/j.ijcm.20210501.12

Received: February 27, 2021; Accepted: March 13, 2021; Published: March 22, 2021

\begin{abstract}
Objective: Hand, foot and mouth disease (HFMD) is an acute infectious disease caused by enterovirus 71 (EV71), Coxsackie virus A16 (CA16) and other enteroviruses. For the treatment of HFMD, there are no recognized specific treatment drugs. In recent years, with the continuous development of traditional Chinese medicine, the clinical use of traditional Chinese medicine in the treatment of HFMD reports is increasing. At present, traditional Chinese medicine is mainly used to disperse wind and heat, clear heat and detoxify, clearing damp and dispel evil, but most of them do not know the exact mechanism of treatment. In this paper, the network pharmacology method was adopted to analyze the main active components and action targets of Sang-Ju-Yin (SJY) and to construct corresponding pathways, and to explore the mechanism of action of SJY in the treatment of HFMD. Methods: The active components of SJY were collected and potential targets were searched by the Traditional Chinese Medicine Systems Pharmacology Database and Analysis Platform (TCMSP). GeneCards platform was used to find disease targets, and a protein interaction network (PPI) was constructed using the STRING platform. Cytoscape 3.6.0 software was used to screen out the key targets. The enrichment analysis of KEGG pathway and gene function analysis (go) was carried out by R language bioconducor package. Results: There were 151 main active components such as quercetin, luteolin and wogonin, and 15 intersection targets were obtained after 78 targets and 100 hand foot mouth disease targets intersected. Key pathways such as TNF signaling pathway, measures and influenza A were obtained by KEGG analysis. Conclusions: The main active components of SJY are quercetin, luteolin, wogonin, kaempferol, aloe emodin, Licochalcone A. It mainly regulates AKT1, Bax, IKBKB, IL-6, STAT3 and other targets, regulates TNF, influenza A and other signaling pathways to inhibit inflammatory response and regulate immune function, so as to achieve the purpose of treating hand foot mouth disease.
\end{abstract}

Keywords: Sang-Ju-Yin, Hand, Foot and Mouth Disease, Network Pharmacology, Target, Signal Pathway

\section{Introduction}

Hand, foot and mouth disease (HFMD) is an infectious disease caused by enterovirus infection. It usually occurs in children under 5 years old. It has strong infectivity and diverse transmission routes. It is a statutory class $\mathrm{C}$ infectious disease in China [1]. According to the incomplete statistics of China's Health Committee and CDC, the mortality of HFMD reported in recent years is $6.46 / 100000-51.00 / 100000$, which is higher in children's infectious diseases and brings heavy burden to families and society. Studies have shown that there are more than 20 kinds of enteroviruses causing hand foot mouth disease, among which coxsackievirus a16 and enterovirus 71 are the most common. The clinical manifestations are infectious fever, accompanied by skin and mucous membrane herpes, ulcers and other typical manifestations in hands, feet, mouth, groin or buttocks [2]. However, there is still a lack of specific drugs for the treatment of HFMD, and broad-spectrum antiviral drugs, such as ribavirin, are widely used in clinic. Traditional Chinese medicine has a good effect on HFMD virus infection by inhibiting inflammation [3], regulating immunity [4] and directly inhibiting virus replication [5-8].

Sang-Ju-Yin (SJY) was published in treatment of epidemic febrile diseases by Wu Jutong in Qing Dynasty. It is composed of 8 medicines including chrysanthemum, bitter platycodon, mint, almond, forsythia, reed root, and raw licorice. It is used for the 
treatment of cough caused by the onset of fever, accompanied by superficial heat symptoms such as floating pulse, slight thirst in mouth and mild body heat. Various medicine compatibility and harmony, in order to evacuate the upper coke wind heat, so that lung qi can be declared, can solve the symptoms, cough [9-11]. The purpose of this study is to screen the bioactive components of SJY, clarify its target, and further clarify the mechanism of network pharmacology in the treatment of HFMD.

\section{Methods}

\subsection{Screening of Active Ingredients and Target}

Traditional Chinese Medicine Systems Pharmacology Database and Analysis Platform (TCMSP http://tcmspw.com/tcmsp.php) consists of 499 Chinese medicines registered in the Chinese Pharmacopoeia, containing 29384 ingredients, 3311 targets and 837 related diseases. The data related to the pharmacokinetic properties of drug screening and evaluation mainly include oral bioavailability (OB), intestinal epithelial permeability (Caco-2), drug-like properties (DL), blood-brain barrier permeability (BBB), Drug half-life (HL), solubility and five principles of drug preparation, etc [12]. This article has used the TCMSP to screen the main active ingredients of SJY. The OB value is set to $\geq 30 \%$ and the DL value is $\geq 0.18$. According to the selected active ingredients, the corresponding component targets are searched from the TCMSP.

\subsection{Disease Target Acquisition and Intersection Target Acquisition}

hand-foot-mouth disease" as the search term to search the Gene Cards database (https://www.genecards.org/) and enter the search term in the search box to get the disease genes in the database. Then the venny 2.1.0 platform hasbeenused to take the intersection of drug targets and disease genes, and get the intersection target of SJY for the treatment of hand, foot and mouth disease.

\subsection{PPI Network Construction}

The STRING database (https://string-db.org/) is the most commonly used protein database, containing more than 5,000 species, more than 20 million proteins, and 2 billion protein interactions, including both direct interactions between proteins and indirect functional correlations between proteins. The database contains not only experimental data, the results of text mining, but also the results of bioinformatics prediction. The target group protein interaction network (PPI network) was constructed using String database, the species were set as "Homosapiens", the lowest interaction threshold was "medium confidence $=0.400$ ", and the protein interaction network map was obtained by hiding scattered nodes.

\subsection{GO Enrichment Analysis and KEGG Enrichment Analysis}

Metascape platform (http://metascape.org/) has powerful functions. It integrates several authoritative databases such as
GO, KEGG, Uniprot, and is used for pathway enrichment analysis of gene targets. It is updated once a month to ensure the accuracy of the data. With the help of this platform, GO and KEGG analysis can be conducted on the candidate targets. Gene Ontology (GO) includes three parts: molecular function (MF), biological process (BP) and cellular component (CC), so as to interpret the anti-tumor biological processes of key targets. Enrichment analysis of Kyoto Encyclopedia of Genes and Genomes (KEGG) was carried out to study the main anti-tumor signaling pathways involved in key targets. According to the relevant targets drawn by the KEGG results, a "pathway-target-complex" network was constructed to further screen the key target genes and active ingredients of SJY in the treatment of hand, foot and mouth disease.

\section{Results}

\subsection{Effective Components and Targets of SJY}

A total of 151 active compounds were screened out according to "OB $\geq 30 \%$ and $\mathrm{DL} \geq 0.18$ " from the TCMSP database, and a total of 278 predicted targets were identified. The number of active ingredients and predicted targets of each drug in the formula was shown in Table 1.

Table 1. Basic information of "compound - active ingredient - target" of SJY.

\begin{tabular}{llll}
\hline TCM & $\begin{array}{l}\text { Number of } \\
\text { compounds }\end{array}$ & $\begin{array}{l}\text { Number of active } \\
\text { ingredients }\end{array}$ & $\begin{array}{l}\text { Number of } \\
\text { Predict targets }\end{array}$ \\
\hline Mulberry leaf & 269 & 29 & 207 \\
chrysanthemum & 359 & 20 & 203 \\
almond & 113 & 19 & 63 \\
Forsythia & 150 & 23 & 205 \\
mint & 164 & 10 & 104 \\
Platycodon & 102 & 7 & 70 \\
Licorice & 280 & 92 & 213 \\
Reed root & 31 & 1 & 27 \\
\hline
\end{tabular}

\subsection{Gene Acquisition and Cross Genes of Hand Foot Mouth Disease}

According to genecards database, 100 HFMD disease genes were obtained. Using the Venny platform, the main active component targets of SJY were crossed with the disease genes of hand foot mouth disease, and 15 targets of SJY for the treatment of hand foot mouth disease were obtained, as shown in Figure 1.

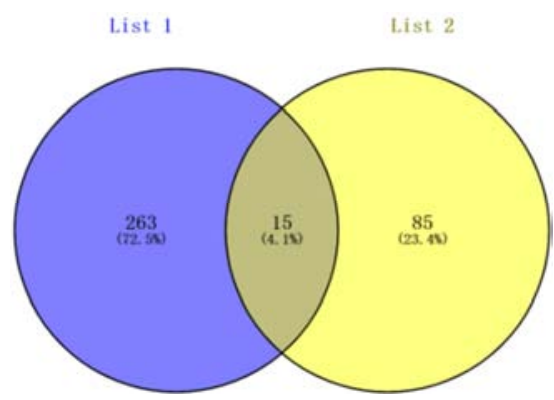

Figure 1. Prediction of the potential target of SYJ against HFMD venny diagram. 


\section{3. "Component-Disease Target" Network Analysis}

Cytoscape 3.7.0 software was used to process the data to obtain information about the effective compounds and targets of Scutellaria baicalensis-Honeysuckle, and establish a network of effective compounds-targets. Quercetin, wogonin, beta-sitosterol, kaempferol, aloe-emodin, licochalcone a, aracidonic acid, acacetin, acacetin, melaleuca A, alpinin, Corymbosin, Epiberberine, beta-carotene, hyperforin, naringenin, luteolin compounds As well as CCL2, HSPB1, AKT1, IL1B, IKBKB, ICAM1, CCND1, CD40LG, IL6, BAX, STAT3, HSPA5, MMP9, EGFR, CRP and other targets play an important role in the network, it may be that SJY plays a clinical role in Chinese medicine As well as the core compounds and targets associated with hand, foot and mouth disease, the results are shown in Figure 2.

\subsection{Analysis of "Component Disease Target" Network}

The effective compounds and action target information obtained by Cytoscape3.7.0 software were processed to establish the effective compounds and action target network, and the results were shown in Figure 2. Quercetin, Wogonin, Beta-Sitosterol, Kaempferol, Aloe-Emodin, Licochalcone A, Arachidonic Acid, Acacetin, Acacia, Paperetin A, Semerin, Corymbosin, Epiberberine, Beta-carotene, Hyperforin, Naringenin, Luteolin compounds CCl2, HSPB1, Akt1, IL1B, IKBKB，ICAM1, CCND1，CD40LG，IL6，Bax, STAT3, HSPA5, MMP9, EGFR, CRP and other action targets play an important role in the network, which may be the core compounds and action targets of Sangju Decoction for the clinical efficacy of traditional Chinese medicine and the association with hand, foot and mouth disease.

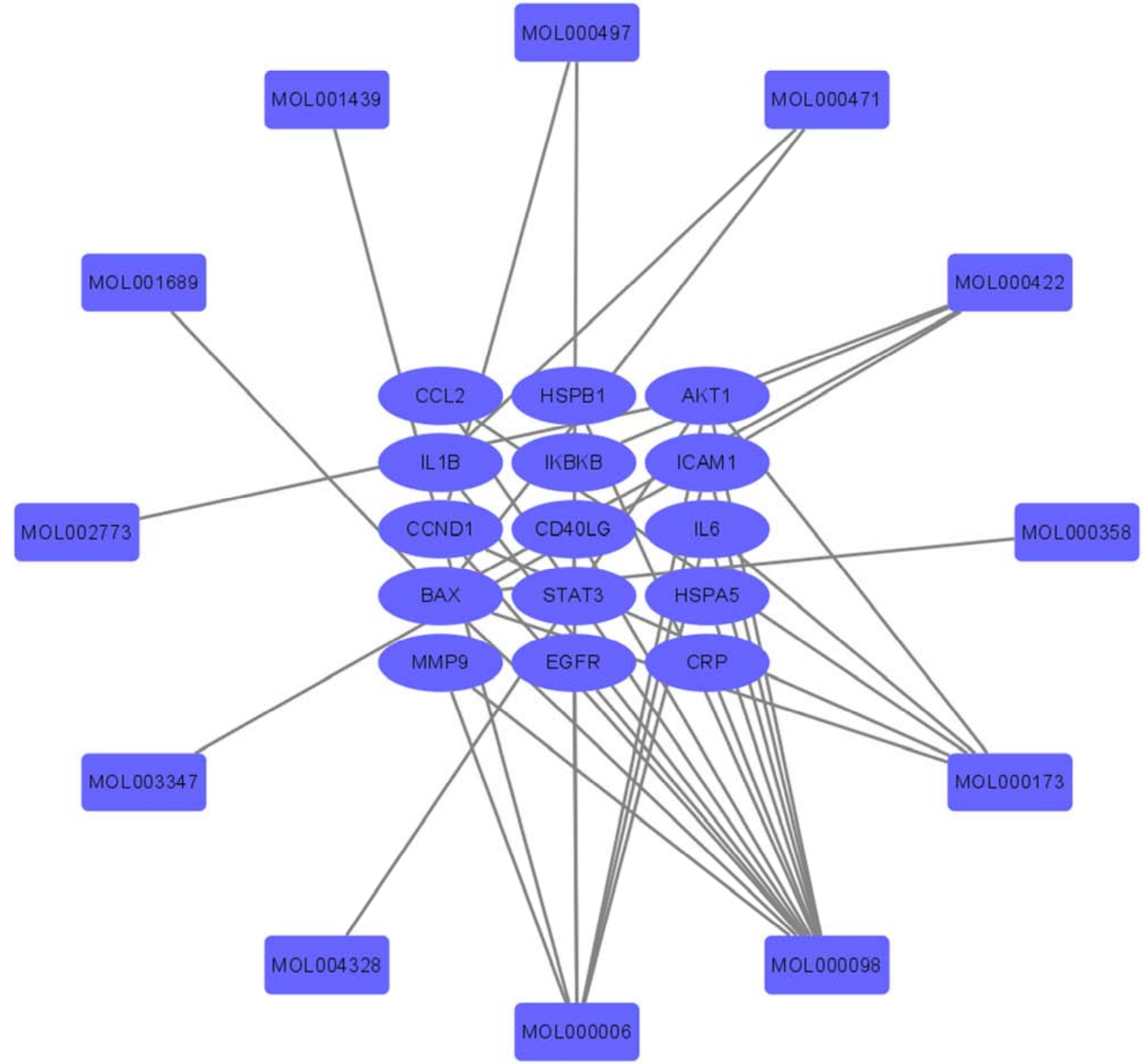

Figure 2. Disease-Compound-Target Network. 


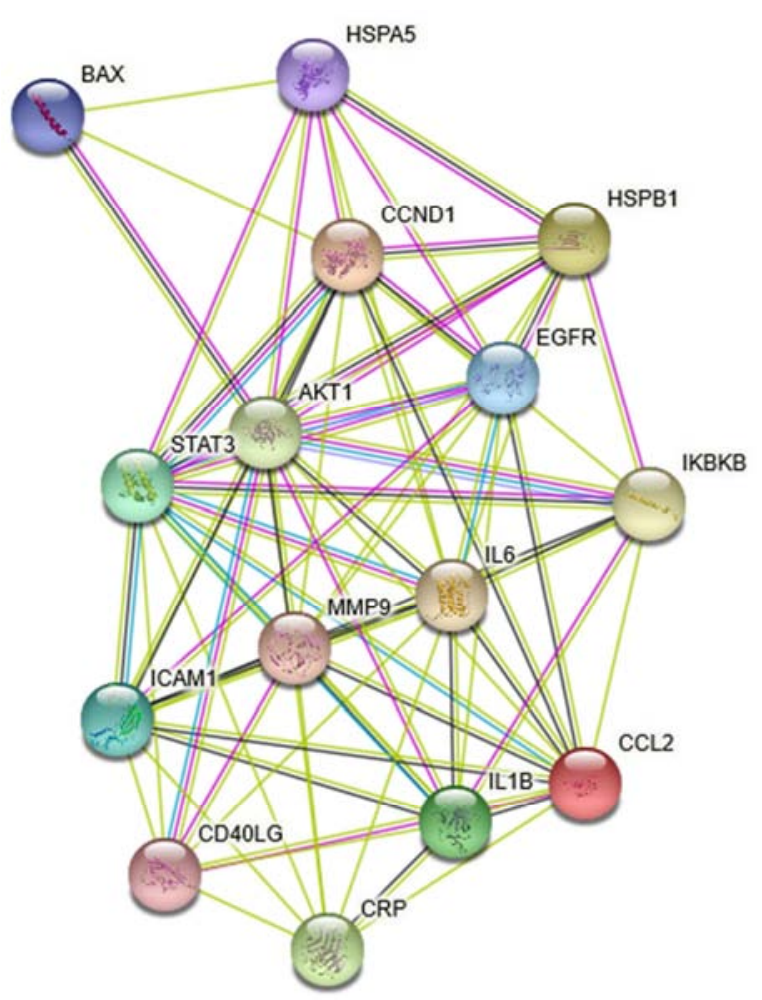

Figure 3. PPI network.

\subsection{PPI Network Construction}

In order to more intuitively understand the mechanism of
Sang Juyin's treatment of hand, foot and mouth disease, the intersection genes of the diseases and drugs obtained above are input into the STRING database to construct a gene function association network. The network contains 15 nodes, 73 edges, and an average node degree of 9.73, as shown in Figure 3.

\subsection{GO Enrichment Analysis of Key Targets and KEGG Pathway Results}

\subsubsection{Key Target GO Enrichment}

GO is the abbreviation of Gene Ontology, and it mainly includes three parts: Biological Process, Cellular Component, and Molecular Function. Organize the target genes of the hands, feet and mouths of the active ingredients of SJY, run the $\mathrm{R}$ language Bioconductor cluster Profiler program package to perform GO enrichment analysis on the target genes, screen and test the gene product annotation process with $\mathrm{P}<0.05$, and follow the $\mathrm{P}$ value Sort from small to large, show the top 10 biological processes, and get Figures 4-6. Figure 4 shows that the common targets in the Biological Process, the main enrichment items are $\mathrm{T}$ cell activation, positive regulation of DNA-binding transcription factor activity, regulation of DNA-binding transcription factor activity and so on. Figure 5 shows that the main enrichment items of its common target in Cellular Component are focal adhesion, cell-substrate adherens junction, and cell-substrate junction. As shown in Figure 6, the main enrichment items of its common target in Molecular Function are cytokine receptor binding, cytokine activity, receptor ligand activity and so on.

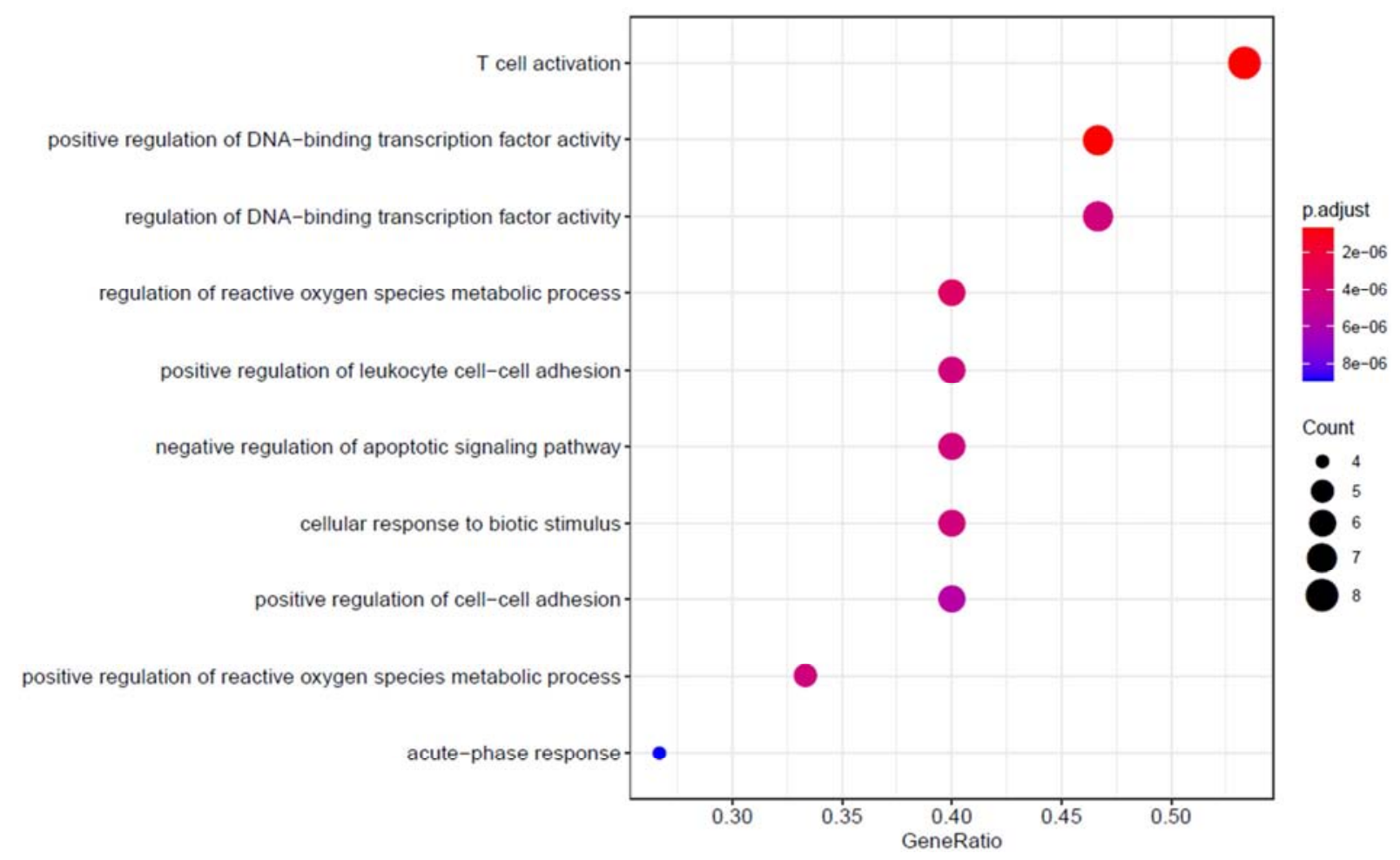

Figure 4. $B P$. 


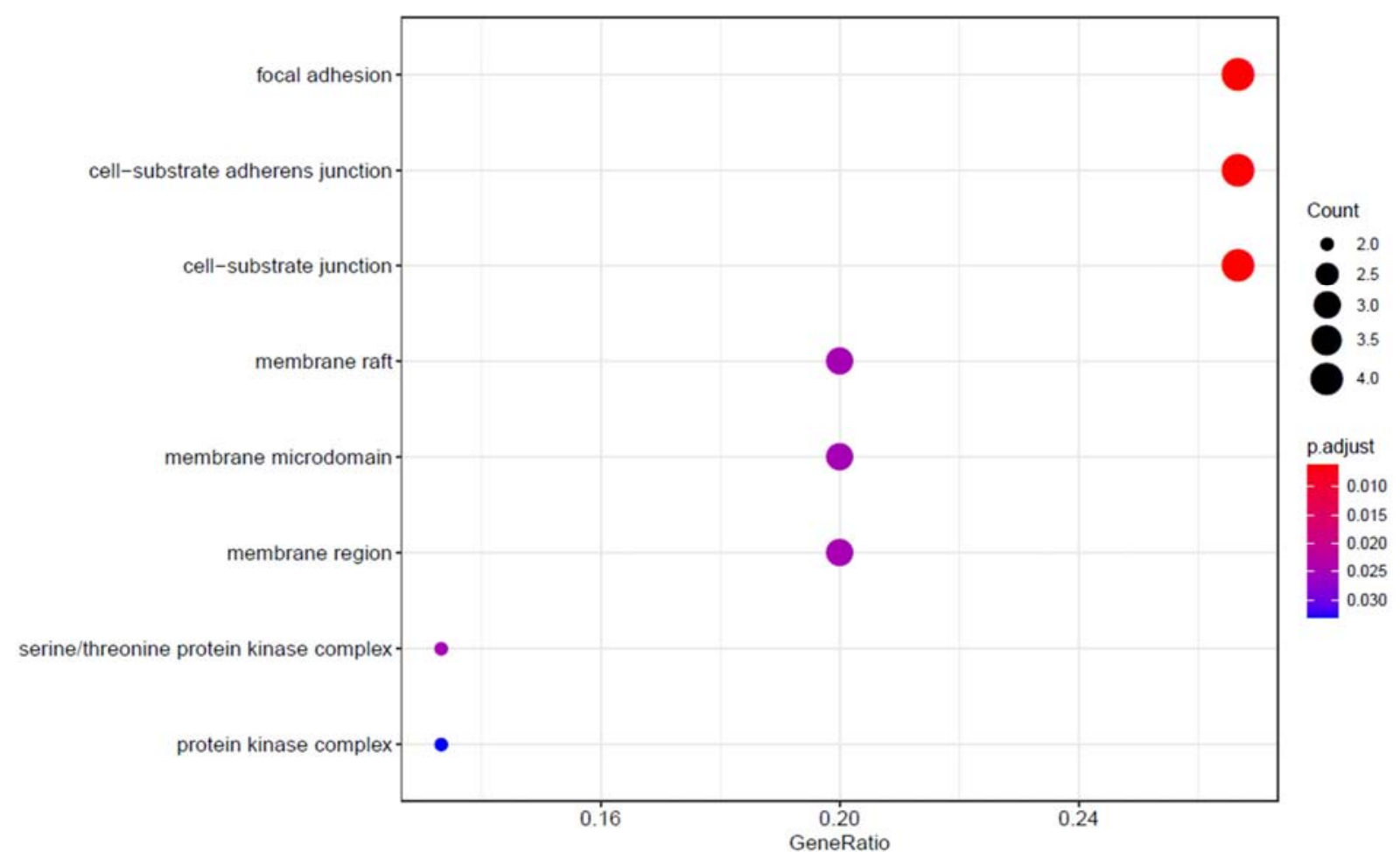

Figure 5. CC.

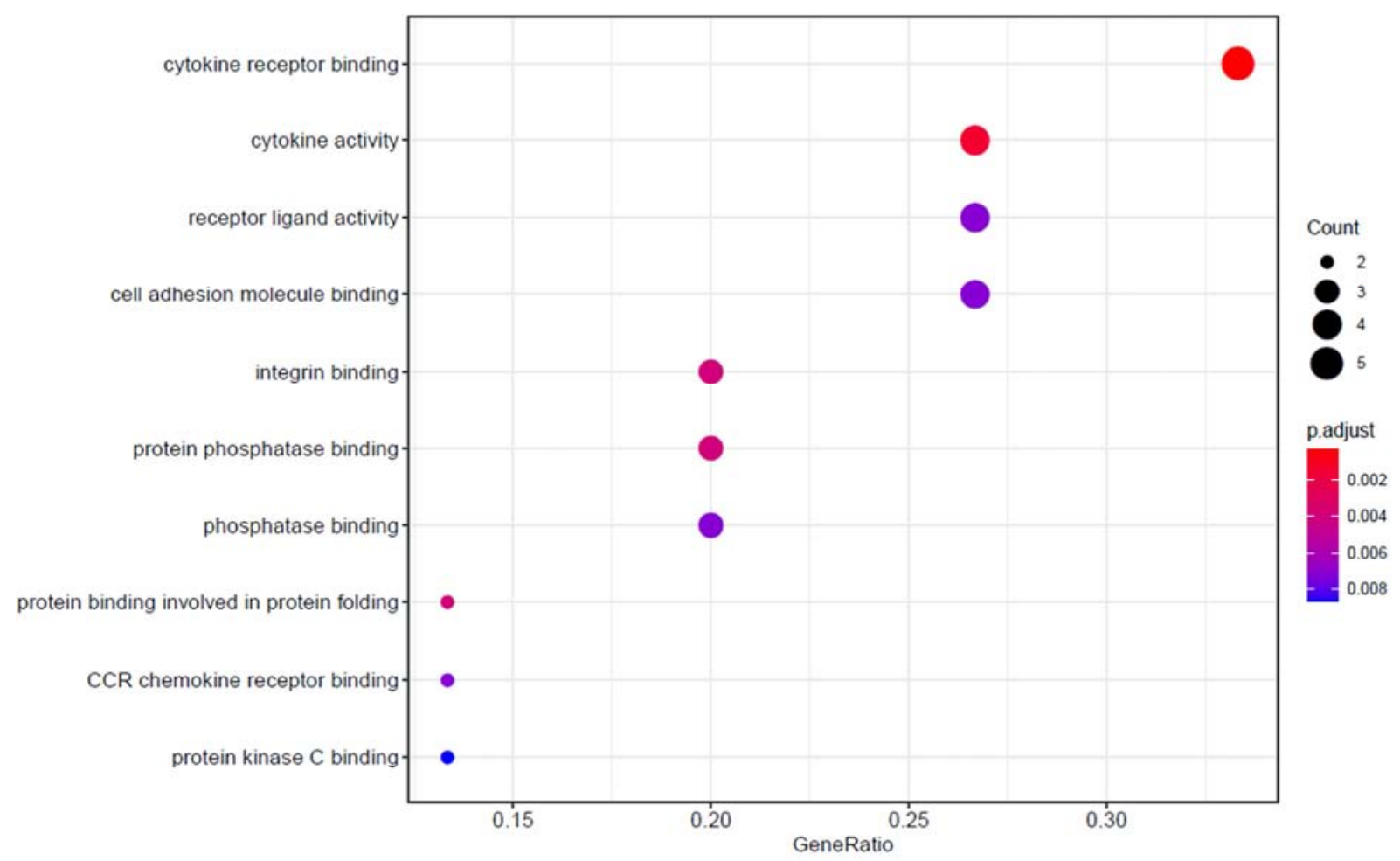

Figure 6. $M F$.

\subsubsection{KEGG Pathway}

$\mathrm{R}$ language Bioconductor clusterprofiler package was used for KEGG pathway enrichment analysis of target genes, and the enriched signal pathways with $\mathrm{P}<0.05$ were selected. According to the $\mathrm{p}$ value, the top 20 signal pathways were visually displayed, as shown in Figure 7. 


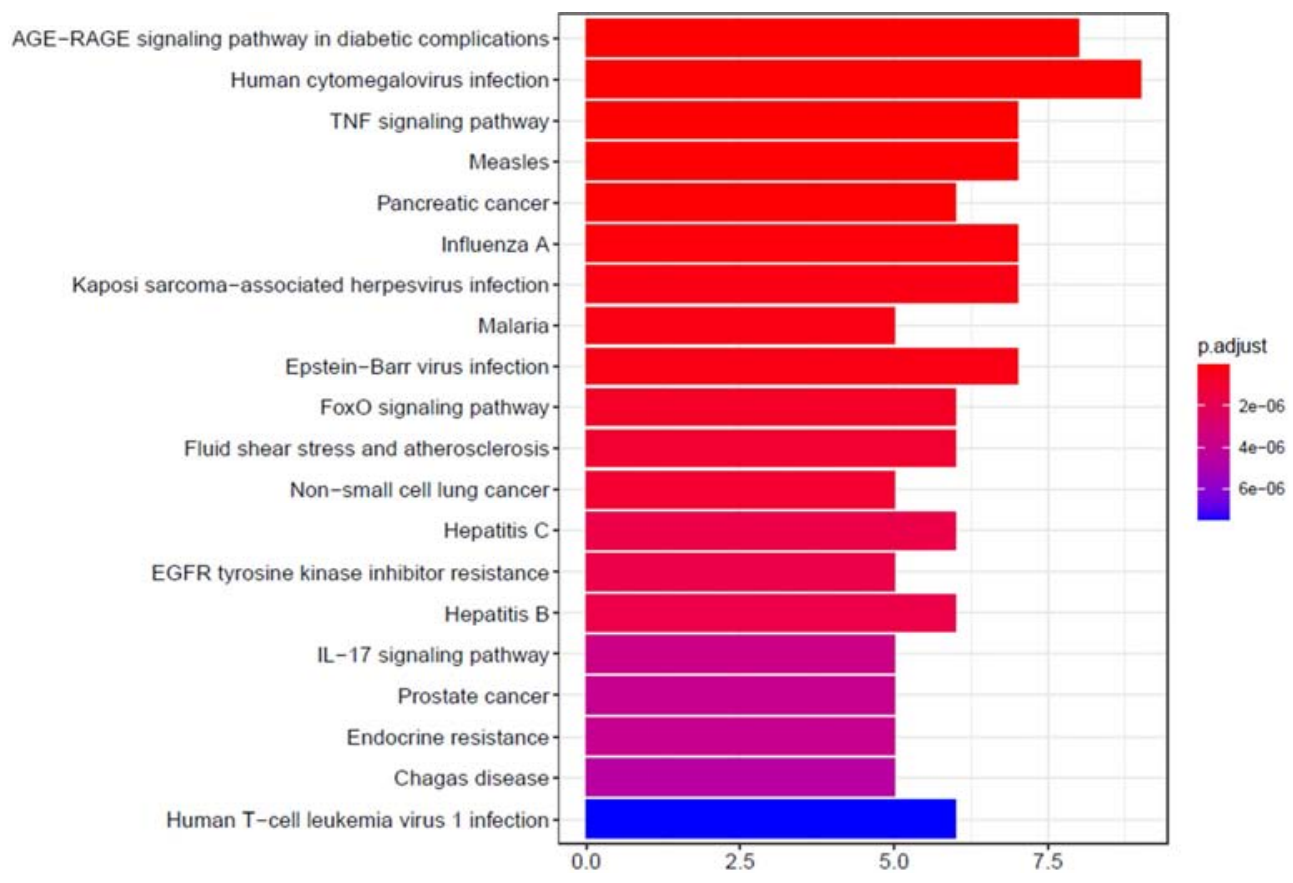

Figure 7. KEGG pathway.

\subsection{Construct a Target-path Diagram}

Import the obtained pathway entries and target information into Cytoscape software, and draw the target- pathway diagram, as shown in Figure 8. The blue represents the pathway and the orange represents the target. The core targets mainly include: BAX, AKT1, MMP9, EGFR, ICAM1, IL1B, IL6, CCND1, IKBKB, CCL2, STAT3, CD40LG.

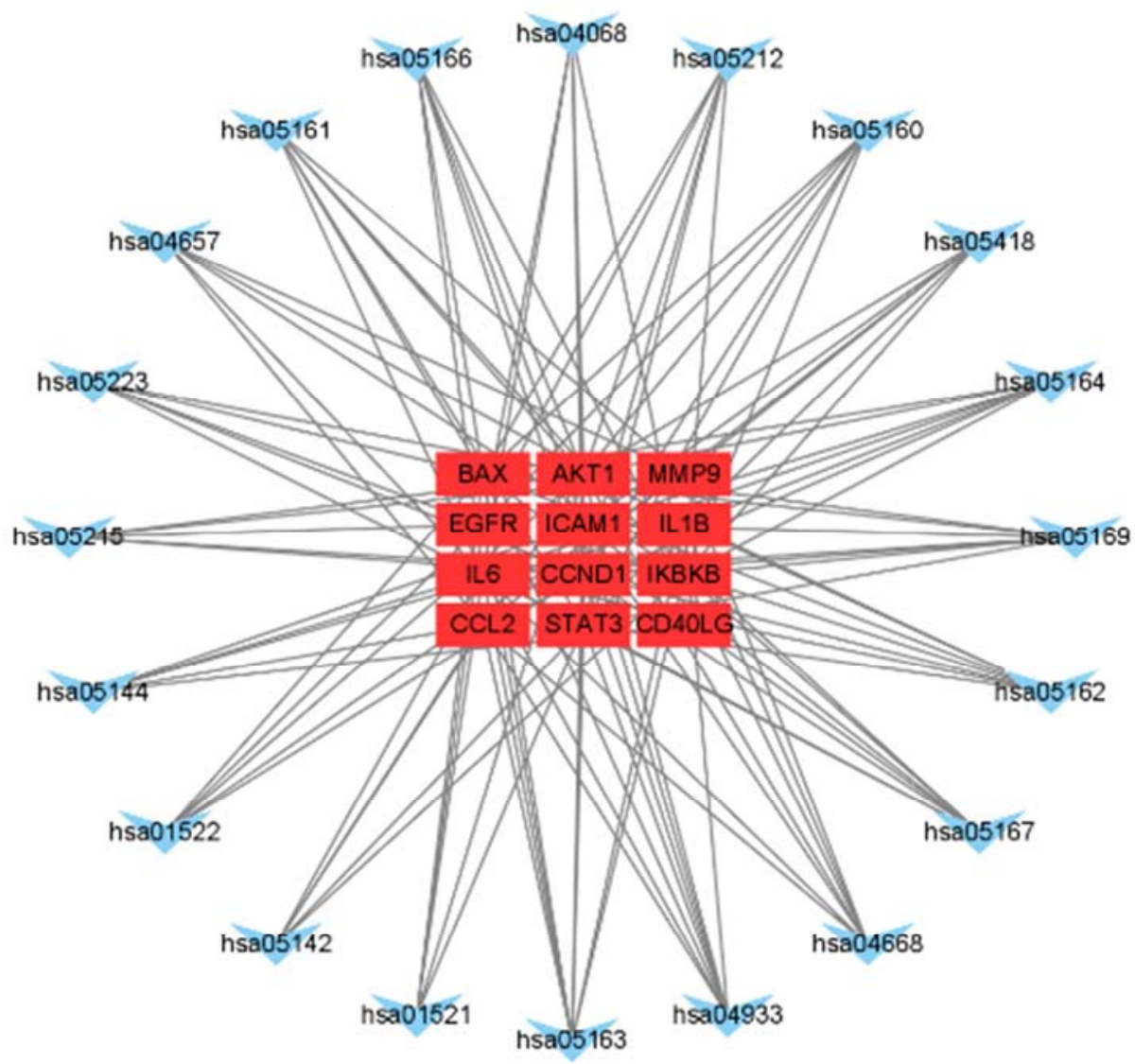

Figure 8. KEGG-target- Network. 


\section{Discussion}

Twelve key chemical components were screened out by the network of "medicinal materials, components, targets and pathways" of Sangju decoctions, including quercetin, wogonin, beta-sitosterol, kaempferol, aloe-emodin, licochalcone A, arachidonic acid, acacetin, beta-carotene, hyperforin, naringenin and luteolin.

Quercetin is the active ingredient of licorice, chrysanthemum, forsythia and mulberry leaf. Wogonin is the active ingredient of forsythia. Beta-sitosterol is the active ingredient of forsythia, mulberry leaf and chrysanthemum. Kaempferol is the active ingredient of forsythia, mulberry leaf, licorice and chrysanthemum active ingredient. Aloe-emodin is the active ingredient of peppermint. Licochalcone $\mathrm{A}$ is the active ingredient of licorice. Arachidonic acid is the active ingredient of mulberry leaves. Acacetin is the active ingredient of peppermint, platycodon, and chrysanthemum. Beta-carotene is the active ingredient of mulberry leaves. Hyperforin is the active ingredient of forsythia. Naringenin is the active ingredient of peppermint, licorice and chrysanthemum. Luteolin is the active ingredient of peppermint, chrysanthemum, platycodon and forsythia.

Studies have shown that quercetin has both anti-infective activity and anti-replication activity. In monolayer cell culture, quercetin can inhibit the infection of cells by a variety of viruses, and can inhibit the replication of viruses in cells. The entry of the virus and receptor complex into the cell must be assisted by calcium ions. Because quercetin can antagonize the calcium ion channel, the virus receptor complex cannot enter the cell, interrupting its life cycle, and causing the death of the virus [13]. It is also the mechanism by which quercetin can better inhibit the virus in vitro.

The main way that kaempferol mediates apoptosis is to change the cell cycle. Kaempferol might cause time- and dose-dependent cell cycle changes in most cells, increasing the number of cells in G0/G1 phase and S phase. In the study of the inhibitory effect on HL-60 cells, it was found that it increased the number of cells in $\mathrm{S}$ phase The effect is more obvious.

A number of studies have found that kaempferol's cell growth inhibitory effect and the effect of mediating cell apoptosis are not as good as quercetin, but compared with other flavonoids such as quercetin, myricetin, isorhamnetin, kaempferol Phenol is the most effective active oxygen free radical scavenger. Kaempferol has a broader role in immune regulation and disease prevention [14].

"Medicinal materials-components-targets-pathways" network of SJY screened a total of 15 key targets, including CCL2, HSPB1, AKT1, IL1B, IKBKB, ICAM1, CCND1, CD40LG, IL6, BAX, STAT3, HSPA5, MMP9, EGFR, CRP [15]. IL6 is a cytokine, a type of interleukin, mainly synthesized by monocytes-macrophages, $\mathrm{T}$ lymphocytes and fibroblasts. It can promote the liver to synthesize acute-phase proteins, activate $\mathrm{T}$ lymphocytes, and induce B lymphocytes [16, 17]. The terminal differentiation of cells makes them immune-competent cells that secrete immunoglobulins $[18,19]$.

\section{Conclusions}

In summary, this study uses the technology and methods of network pharmacology to search for active ingredients and potential targets of traditional Chinese medicine in SJY, as well as hand-foot-mouth disease-related targets. Among them, there are 15 common drug-disease targets. The target is subjected to GO biological process and KEGG signal pathway enrichment analysis, and it is predicted that the main active ingredients of SJY are quercetin, luteolin, wogonin, kaempferol, aloe-emodin, licochalcone A, which are mainly regulated by AKT1, BAX, IKBKB, IL -6, STAT3 and other targets, regulate TNF, Influenza A and other signal pathways to inhibit inflammation and regulate immune function, so as to achieve the purpose of treating hand, foot and mouth disease.

\section{References}

[1] China NHCO. (2018). Hand, Foot and Mouth Disease Diagnosis and Treatment Guidelines (2018 Edition). 11: 161.

[2] Baoqiang Q. (2016). Research progress on the etiology of hand, foot and mouth disease. Guangxi Medical Journal 38: 698-700.

[3] Xiao ZCXC. (2015). Antiviral effects of Reduning injection against enterovirus 71 and possible mechanisms of action. Chin J Nat Med 13: 881.

[4] Li M, Yang X, Guan C, Wen T, Duan Y, Zhang W, Li X, Wang Y, Zhao Z and Liu S. (2018). Andrographolide sulfonate reduces mortality in enterovirus 71 infected mice by modulating immunity. Int Immunopharmacol 55: 142-150.

[5] Yeo S, Yeo S, Song JH, Song JH, Hong E, Hong E, Lee B, Lee B, Kwon YS, Kwon YS, Chang S, Chang S, Kim SH, Kim SH, Lee SW, Lee SW, Park J, Park J, Ko H and Ko H. (2015). Antiviral effects of Phyllanthus urinaria containing corilagin against human enterovirus 71 and Coxsackievirus A16 in vitro. Archives of pharmacal research 38: 193-202.

[6] Chen SG, Cheng ML, Chen KH, Horng JT, Liu CC, Wang SM, Sakurai H, Leu YL, Wang SD and Ho HY. (2017). Antiviral activities of Schizonepeta tenuifolia Briq. against enterovirus 71 in vitro and in vivo. Sci Rep 7: 935.

[7] Jianhui D. Effectiveness Analysis of Pediatric Resuqing Granule in Hand, Foot and Mouth Disease. Systems Medical 5: 90-92.

[8] Genlin Z, Shuhua L and Feng K. (2017). Clinical observation of Resuqing Granules for children combined with Ribavirin in the treatment of hand-foot-mouth diseases. China Modern Medicine 24: 132-134.

[9] Ruitang Z. (2004). Discussion on the characteristics and application of SJY prescription. CLINICAL JOURNAL OF TRADITIONAL CHINESE MEDICINE 1: 27-28.

[10] Long-ying Z. (2007). Clinical Observation of Wind-Dispersing and Throat-Clearing, Lung-Arresting and Cough-Relieving Therapy for Laryngeal Cough. SHANGHAI JOURNAL OF TRADITIONAL CHINESE MEDICINE 41: 28-29. 
[11] Chunnan H. (2000). Examples of new clinical uses of SJY. JOURNAL OF PRACTICAL TRADITIONAL CHINESE MEDICINE: 42.

[12] Ru J, Li P, Wang J, Zhou W, Li B, Huang C, Li P, Guo Z, Tao W, Yang Y, Xu X, Li Y, Wang Y and Yang L. (2014). TCMSP: a database of systems pharmacology for drug discovery from herbal medicines. Journal of cheminformatics 6: 1-6.

[13] Wu SN, Chiang HT, Shen AY and Lo YK. (2003). Differential effects of quercetin, a natural polyphenolic flavonoid, on L-type calcium current in pituitary tumor (GH3) cells and neuronal NG108-15 cells. J Cell Physiol 195: 298-308.

[14] Xiaoqing L, Ao C, Yi L and Jun H. (2017). Research progress in pharmacological effects of kaempferol, research on trace elements and health. Studies of Trace Elements and Health 42: 61-62.

[15] Xiao-Long Y, Yan S, Yu-Qin Q, Wang Y and Chang-Gui L. (2004). Effect of NaI on cytokines secretion in cultured human thyrocytes. Immunol J 2: 124-125.
[16] Kraakman MJ, Kammoun HL, Allen TL, Deswaerte V, Henstridge DC, Estevez E, Matthews VB, Neill B, White DA, Murphy AJ, Peijs L, Yang C, Risis S, Bruce CR, Du XJ, Bobik A, Lee-Young RS, Kingwell BA, Vasanthakumar A, Shi W, Kallies A, Lancaster GI, Rose-John S and Febbraio MA. (2015). Blocking IL-6 trans-signaling prevents high-fat diet-induced adipose tissue macrophage recruitment but does not improve insulin resistance. Cell Metab 21: 403-416.

[17] Haidar M, Asselbergh B, Adriaenssens E, De Winter V, Timmermans JP, Auer-Grumbach M, Juneja $M$ and Timmerman V. (2019). Neuropathy-causing mutations in HSPB1 impair autophagy by disturbing the formation of SQSTM1/p62 bodies. Autophagy 15: 1051-1068.

[18] Vendredy L, Adriaenssens E and Timmerman V. (2020). Small heat shock proteins in neurodegenerative diseases. Cell Stress Chaperones 25: 679-699.

[19] Pengbo Y and Shuhua L. (2011). The application of interleukin-6 in clinical diagnosis of Parkinson disease. CHINESE JOURNAL OF NEUROIMMUNOLOGY AND NEUROLOGY 18: 45-47. 